\title{
CHINA EN 2018: CAMBIOS, REAJUSTES INTERNOS Y ENFRENTAMIENTO COMERCIAL CON ESTADOS UNIDOS
}

\author{
Marisela Connelly \\ El Colegio de México
}

\section{INTRODUCCIÓN}

El año 2018 ha representado un gran reto para el gobierno de la República Popular China; la profundización y agilización de la reforma económica siguen siendo su prioridad; la necesidad de regulación de las diferentes entidades económicas y financieras se hace cada vez más compleja a medida que avanza la economía a una en que las fuerzas del mercado tienen un papel más importante y en que, al mismo tiempo, el Estado chino se ve a sí mismo como el árbitro de todo el proceso de cambio. El papel del líder máximo se fortaleció con las decisiones tomadas en la Décimo Tercera Asamblea Popular Nacional de marzo, que eliminan el límite para la permanencia en los puestos de presidente y vicepresidente del país. Lo mismo sucede con la reorganización institucional que se llevó a cabo, tendiente a hacer más eficaces y funcionales en sus respectivas carteras, a los ministerios y agencias del gobierno.

En el escenario internacional China sigue proyectando una imagen de líder en los diferentes organismos que preside y donde participa, como la Organización de Cooperación de Shanghái y el grupo de los BRICS. ${ }^{1}$ Ha mejorado su relación con los países del Sudeste de Asia y con Japón y ha tenido una participación activa en las negociaciones sobre la península coreana, en tanto que los problemas con Estados Unidos se han incrementado al grado de entrar en conflicto comercial, con la imposición de tarifas a sus respectivos productos por parte de ambos.

\footnotetext{
${ }^{1}$ Brasil, Rusia, India, China y Sudáfrica.
} 
El liderazgo chino ha continuado con su política tendiente a aislar a Taiwán, quitándole aliados diplomáticos que por décadas le habían sido leales a la isla, como son los casos de El Salvador y República Dominicana. También ha intentado ganarse a los taiwaneses al otorgarles mayores prerrogativas durante su permanencia en el territorio continental.

\section{POLÍTICA}

Del 5 al 20 de marzo se llevó a cabo la Décimo Tercera Sesión de la Asamblea Popular Nacional (APN). El presidente Xi Jinping resaltó en su discurso varios puntos importantes: en particular, recalcó que los poderes del Estado pertenecen al pueblo; definió la Dictadura Democrática Popular como dirigida por los obreros y basada en la alianza obrero-campesina; el progreso, basado en la estabilidad; el Partido Comunista de China como líder y fuerza capaz de llevar al país por la senda del rejuvenecimiento; la necesidad de trabajar para que Hong Kong y Macao se asuman como parte de China y su gente tenga un sentido de pertenencia y, por tanto, la necesidad de fomentar el patriotismo. Señaló la imposibilidad de que alguna parte de China dejara de serlo, lo que muestra una posición dura respecto a cualquier intento de declararse independientes. En el aspecto internacional, Xi mostró su visión sobre la posición de China, asegurando que el crecimiento económico del país no implica amenaza para otros, ni tampoco se desarrolla a expensas de otros, y por tanto no pretende enfrentarse al país hegemónico, léase Estados Unidos. Insistió en que China seguirá ayudando a los países en vías de desarrollo y a los países que están sumergidos en la guerra (South China Morning Post, 20 de marzo de 2018).

En su informe, el primer ministro Li Keqiang mostró con cifras el incremento del Producto Interno Bruto en el consumo y los servicios. Destacó que China tiene una inversión en investigación y desarrollo que la coloca en segundo lugar en el ámbito mundial.

Los legisladores votaron para eliminar los límites de permanencia en los puestos de presidente y vicepresidente de la RPCh. Anteriormente se tenía un periodo de cinco años, renovable una sola vez. Sólo 2 de los 2964 diputados votó en contra de las revisiones constitucionales, 3 se abstuvieron, 1 boleta fue invalidada y 16 diputados no estuvieron presentes; por tanto, la modificación quedó aprobada. Los puestos de primer ministro, viceprimer ministro y presidente de la ANP continúan con los límites a sus periodos. No hubo discusión ni debate. 
Xi Jinping reafirmó su poder al aceptarse añadir en la Constitución su pensamiento sobre el socialismo con características chinas para una nueva era. Además, Xi juró públicamente su adherencia a la Constitución. Así, Xi fue electo como presidente de la República y como presidente de la Comisión Central Militar (Strait Times, 17 de marzo de 2018). El lugar del Partido Comunista de China queda asentado en la Constitución al afirmarse que su liderazgo es el rasgo fundamental del "socialismo con características chinas". Wang Qishan quedó como vicepresidente.

Xi Jiping ha logrado imprimir su sello en la Constitución, fortaleciendo no sólo su liderazgo sino también la legitimidad del PCCh, institucionalizando su posición y haciendo de nuevo tenue la línea entre el Estado y el Partido. El razonamiento es que en la actual etapa que vive la reforma económica, los problemas sociales que ha generado y la defensa de la posición de China en el escenario internacional requieren un líder y un partido fuertes.

\section{RESTRUCTURACIÓN INSTITUCIONAL}

Los cambios forman parte de un plan más amplio del PCCh para profundizar la reforma del mismo partido y la de las instituciones estatales, así como el fortalecimiento de algunos ministerios claves en el proceso de reforma, concentrando en ellos funciones que anteriormente habían estado dispersas en diferentes ministerios y agencias. La nueva Comisión de Estabilidad Financiera y Desarrollo de China dentro del Consejo de Estado tiene como objetivo supervisar las actividades financieras y proveer de guía a futuras reformas y restructuraciones. La creación de esta comisión se hizo necesaria ante los fracasos en materia de regulación de 2016 y 2017.

También se crearon la Comisión Regulatoria de Bancos y Seguros de China; la Comisión de Seguridad Interna, para proteger la ley y el orden, controlar la información de seguridad interna, prever riesgos y formular la política anti culto religioso; el Ministerio de Recursos Naturales, el cual nace del Ministerio de Tierras y Recursos pero absorbe funciones de la Comisión Nacional de Reforma y Desarrollo, de Planificación Urbana del Ministerio de Construcción y manejo de tierras del Ministerio de Agricultura; el Ministerio de Ecología y Medio Ambiente, que reemplaza al Ministerio de Protección Ambiental, asumiendo responsabilidades que estaban dentro de la Comisión Nacional de Reforma y Desarrollo, del Ministerio de Recursos Hídricos, del Ministerio de Agricultura y del Ministerio de Tierras y 
Recursos; todo ello con el fin de controlar la contaminación y de mejorar el medio ambiente, que es una de las demandas del pueblo chino; el Ministerio de Recursos Naturales, encargado de la protección y desarrollo de los recursos, tales como bosques, humedales, pastizales y recursos marítimos; el Ministerio de Agricultura y Asuntos Rurales, que combina la supervisión de la agricultura con el desarrollo rural (China desea eliminar la pobreza extrema que se concentra en áreas rurales, y fomentar la producción agrícola mediante el uso eficiente de la tierra y de la tecnología); el Ministerio de Asuntos de Veteranos, para mejorar el servicio y el sistema administrativo del personal militar desmovilizado para prevenir la insatisfacción en el Ejército de Liberación Popular (Xinhua, 13 de marzo de 2018); el Ministerio de Emergencias, encargado de asuntos de seguridad laboral y desastres naturales, como incendios, inundaciones, sequías, huracanes y terremotos; el Ministerio de Cultura y Turismo, para fortalecer el poder suave de China y difundir su cultura; La Comisión Nacional de Salud, para coordinar y llevar a cabo la reforma en salud, establecer un sistema de medicinas básicas a escala nacional, y supervisar y administrar la salud pública y las emergencias en dicha área.

Ahora bien, dos pequeños grupos dirigentes se convirtieron en comisiones: la Comisión Central para la Profundización y Ampliación de la Reforma, y la Comisión Central sobre Finanzas y Economía.

El Consejo de Estado quedó constituido por 26 ministerios y comisiones, además de la Oficina General del Consejo de Estado. Ministerios: de Asuntos Exteriores, de Defensa Nacional, de la Comisión de Reforma y Desarrollo; de Educación, Ciencia y Tecnología, de la Comisión Estatal de Asuntos Étnicos, de Seguridad Pública, de Seguridad del Estado, de Asuntos Civiles, de Justicia, de Finanzas, de Recursos Humanos y Seguridad Social, de Medio Ambiente Ecológico, de Vivienda y Desarrollo Urbano Rural, de Transporte, de Recursos Acuíferos, de Asuntos Agrícolas y Rurales, de Comercio, de Cultura y Turismo: la Comisión Nacional de Salud, la de Asuntos de Veteranos, la de Manejo de Emergencias, la del Banco Popular de China y la de la Oficina Nacional de Auditoría (Xinhua, 13 de marzo de 2018). 


\section{NUEVOS VICEMINISTROS}

Han Zheng, como viceprimer ministro ejecutivo, tomó el lugar de Zhang Gaoli; su tarea consiste en supervisar a las agencias económicas. Zheng desempeñó el puesto de alcalde de Shanghái por diez años y fue primer secretario del PCCh durante cinco años.

Liu He, asesor económico y persona de confianza de Xi Jinping, estudió en la Harvard Kennedy School. En su nuevo puesto, Liu se encarga de supervisar las relaciones comerciales internacionales y de las negociaciones con Estados Unidos; también atiende la política tecnológica.

Sun Chunlan es la encargada de Educación y Salud. Sus estudios los realizó a distancia: el bachillerato en economía en la Universidad de Liaoning y su carrera en Ciencia Política en la Escuela Central del Partido. Fue secretaria de la Municipalidad de Tianjin. Desde 2014 estuvo en el Departamento de Frente Unido del Partido Comunista Chino.

$\mathrm{Hu}$ Chunhua combina en su puesto funciones de negociación comercial internacional y alivio a la pobreza. Fue secretario del Partido en Guangdong, de 2012 a 2017.

Liu Kun es el nuevo ministro de Finanzas. No es muy conocido. Trabajó durante treinta años en el gobierno provincial de Guangdong; en 2010 se convirtió en vicegobernador del Banco Fiscal Provincial. En 2012 se mudó a Beijing tras ser nombrado viceministro de Finanzas. La reforma fiscal se estancó en 2014; Liu tendrá que agilizarla y establecer el nuevo sistema de relaciones fiscales entre el centro y las localidades.

Yi Gang fue nombrado director del Banco Popular de China por el primer ministro Li Keqiang el 19 de marzo. Es una persona muy preparada e inteligente que conoce el trabajo cotidiano del banco. Tiene un PhD en economía por la Universidad de Illinois.

Guo Shuqing fue nombrado secretario del PCCh en el Banco Popular de China. Guo trabajó en la provincia de Shandong y en 2017 fue nombrado director de la Comisión Reguladora Bancaria de China (Naugthon, 2018a).

Como puede observarse, Xi Jinping formó un grupo de colaboradores de primer nivel, que hábilmente ha colocado en puestos clave. Todos ellos están comprometidos con la continuación de la reforma económica orientada al mercado. El propósito de crear nuevos ministerios y de eliminar otros, fusionar funciones y restarle poder a la Comisión Nacional 
de Reforma y Desarrollo, tiene como objetivo hacer más eficiente el trabajo, facilitar la trasmisión de decisiones de arriba hacia abajo, delimitar funciones y fortalecer la rendición de cuentas.

\section{ECONOMÍA}

La actividad económica de China se encuentra en un estado sano, con un crecimiento de $6.9 \%$ en 2017, y de $6.8 \%$ en el primer cuarto de 2018. A diferencia de 2017, las exportaciones no fueron el motor de crecimiento en el primer cuarto de este año, sino el consumo. Software y servicios de tecnología informática están creciendo a doble dígito, y contribuyeron con 1.1\% al crecimiento en el primer cuarto de 2018 (World Bank, 2018).

El crecimiento de la inversión ha bajado debido a causas estructurales que privilegian el consumo sobre la inversión. También han influido en los esfuerzos por reducir la sobrecapacidad industrial y los riesgos financieros. La balanza en cuenta corriente sigue bajando a medida que las exportaciones adquieren un papel menos relevante en el crecimiento del Producto Interno Bruto (PIB). La regulación monetaria ha mostrado resultados; el nivel de deuda corporativa se estabilizó por debajo de 160\% del PIB en 2017. En materia fiscal, creció el gasto de los gobiernos locales, pero se han tomado medidas para que no salga de control. Se espera que el crecimiento en 2018 sea de $6.5 \%$, teniendo en cuenta que la actividad económica avanza a paso más lento debido a varios factores: mayor control en materia de política monetaria y fiscal, crecimiento moderado del comercio global, continuación de las reformas para enfrentar la sobrecapacidad industrial, y problemas de medio ambiente. Los principales riesgos para la economía los constituyen la deuda corporativa y las tensiones comerciales.

El consumo se vio fortalecido luego de darse una alza en el ingreso y una baja tasa de desempleo. El comercio en línea se incrementó; además, bajó el Impuesto al Valor Agregado (IVA) en varios productos.

En marzo, durante la Décimo Tercera Sesión de la ANP se tomaron medidas para apoyar la economía por medio del mejoramiento de la infraestructura y de mayores regulaciones; también, para restructurar a las empresas estatales y hacerlas más competitivas globalmente; 
asimismo, para abrir más el sector financiero, la manufactura de autos, barcos y aviones a la inversión extranjera.

En el segundo cuarto de 2018, el crecimiento del PIB fue de $6.7 \%$ en relación con el mismo periodo del año anterior. Si las tensiones persisten entre China y Estados Unidos, las presiones sobre la economía china se incrementarán; el desempleo continuó controlado con tasas inferiores a 5\%. En la reunión del politburó del $\mathrm{PCCh}$, los líderes chinos decidieron seguir controlando los precios de los bienes raíces y continuar con una política fiscal proactiva y una política monetaria prudente para la segunda mitad de 2018; también, profundizar la reforma estructural del lado de la oferta; cortar el exceso de capacidad, mayor inversión en infraestructura, y una baja en los gastos y costos de las empresas.

El nivel de consumo constituyó el 78.5\% del total del crecimiento del PIB en la primera mitad de 2018. Por otro lado, China está abriendo más sectores a la inversión extranjera, como transporte, finanzas, servicios profesionales, infraestructura, energía, recursos y agricultura, con diferentes niveles de participación (Bin Zhao, 2018).

China, pues, sigue poniendo énfasis en el desarrollo de las Zonas de Libre Comercio (ZLC). En Guangdong se estableció una en 2014 y otra en 2017; el valor de las exportaciones y las importaciones sobrepasó los 142.4 mil millones de dólares. El presidente Xi Jinping visitó Guangdong para dar un discurso por los 40 años de la puesta en práctica de la reforma económica. El 16 de octubre, el Consejo de Estado dio a conocer un plan para establecer la ZLC más grande en Hainan (Global Times, 26 de octubre de 2018; China Daily, 26 de octubre de 2018, Xinjingbao, 26 de octubre de 2018).

En julio China redujo las tarifas de 1449 productos, de un promedio de 15.7 a $6.9 \%$. Las tarifas de importación de vehículos bajaron de 21.5 a $13.8 \%$, y las de autopartes de 10.2 a 6\%. China redujo también las tarifas de Nación más Favorecida a 1585 artículos a partir del 1 de noviembre.

En el tercer trimestre de 2018 el PIB creció 6.5\%, en septiembre la inflación se ubicó en 2.5\%. Según el Banco Mundial; China es la segunda economía más grande del mundo, detrás de Estados Unidos y delante de Japón. Según el Fondo Monetario Internacional, China tenía las mayores reservas en moneda extranjera hasta diciembre de 2017, con 3140 mil 
millones de dólares. Actualmente China ha firmado 17 acuerdos de libre comercio, 13 están en negociación y 10 más en observación.

\section{CHINA Y EsTADOS UNIDOS}

La administración de Donald Trump ha lanzado una guerra comercial en diferentes frentes, imponiendo tarifas sobre acero, aluminio y paneles solares procedentes de países como Canadá y México, la Unión Europea y Japón. Pero con China la situación se volvió crítica porque afecta a un mayor número de productos y a compañías que se basan en cadenas de producción globales. Las mismas compañías estadounidenses se ven afectadas con estas medidas. El razonamiento de Trump va en el sentido de cambiar la situación que ha prevalecido en décadas pasadas, donde China ha obtenido grandes ganancias a costa de Estados Unidos, tanto por prácticas comerciales injustas como por el robo de propiedad intelectual estadounidense. El gobierno de Trump también está poniendo obstáculos para la obtención de visas a ciudadanos chinos y a la inversión china. Trump quiere obligar a China a que abra más sus mercados a las compañías estadounidenses y a eliminar el requerimiento, para esas compañías, de proporcionarle tecnología. Las medidas tomadas por la actual administración de Estados Unidos están afectando directamente a compañías que importan, por ejemplo, partes para automóviles desde China.

La imposición de tarifas discriminatorias a productos chinos ha llevado a que el gobierno chino siga también esta línea, imponiendo tarifas a importaciones procedentes de Estados Unidos. El 17 de abril de 2018, el gobierno chino anunció tarifas de 178.6\% sobre importaciones de sorgo estadounidense.

En mayo, una delegación encabezada por el secretario de Tesoro Mnuchin, y el representante de comercio Robert Lighthizer, llegó a Beijing con una lista de demandas que fueron inaceptables para China. En el mismo mes, una delegación china encabezada por el viceprimer ministro Liu He fue a Washington para entablar negociaciones con el grupo estadounidense presidido por Mnuchin. La parte china ofreció incrementar la compra de productos estadounidenses y reducir el déficit comercial. Trump vetó el acuerdo.

China impuso a los autos de Estados Unidos una tarifa de 40\%, en tanto que bajó la de los autos procedentes de Corea del Sur, Japón y Europa, de 25 a 15\% (Naughton, 2018b). 
En junio se llevó a cabo otra ronda de negociaciones entre funcionarios estadounidenses y chinos en la ciudad de Beijing. No hubo resultados satisfactorios.

El 15 de junio, el gobierno estadounidense impuso una tarifa de $25 \%$ a las importaciones chinas por un valor de 50 mil millones de dólares. China respondió con tarifas a 659 productos estadounidenses, también con valor de 50 mil millones de dólares. El 22 y 23 de agosto se llevaron a cabo negociaciones entre el subsecretario del Tesoro de Estados Unidos, Davis Malpass, y el viceministro de Comercio Wang Shuowen. No hubo acuerdos (Glaser y Flaherty, 2018). En septiembre Trump impuso tarifas sobre 200 mil millones de dólares a productos chinos, la cifra total con lo que llegaba a 250 mil millones de dólares. Las tarifas serían en un principio de $10 \%$, posteriormente Trump anunció que subirían a $25 \%$ a partir del 1 de enero de 2019. Como respuesta, China canceló las pláticas que estaban programadas para discutir el problema. Además, impuso tarifas a productos estadounidenses con valor de 60 mil millones de dólares.

Este enfrentamiento comercial daña a las dos economías, pues interfiere con el crecimiento de China y debilita la economía de Estados Unidos, que todavía siente los estragos de la crisis económica global. También impacta en el crecimiento del comercio global.

Además del conflicto comercial, el gobierno de Estados Unidos ha aplicado sanciones a la compañía de telecomunicaciones ZTE por violar las sanciones impuestas por este país contra Irán y Corea del Norte. El 16 de abril, el Departamento de Comercio le prohibió hacer negocios con compañías de Estados Unidos por un periodo de siete años. El 13 de mayo, en un comunicado, Trump prometió ayudar a la ZTE; esto debido a una petición del mismo Xi Jinping. Pero el Congreso inmediatamente tomó medidas para evitarlo, y en una votación del Comité Bancario del Senado se aprobó por 23 votos a favor y 2 en contra continuar con las sanciones a la ZTE. Finalmente, el 7 de junio Ross anunció un acuerdo con ZTE en el que este último pagaría 1.4 mil millones de dólares. El 13 de julio, una vez que la ZTE pagó la cantidad especificada, se removieron las sanciones (Glasser y Flaherty, 2018).

En el mes de septiembre el gobierno chino hizo público un documento en el que explica cómo se ha desarrollado la relación comercial con Estados Unidos. Dice que el respeto a las reglas y contratos ha sido el fundamento de la economía de mercado y del orden internacional desde el inicio de los tiempos modernos. Afirma que la administración de Estados Unidos no 
respeta el orden en la gobernanza global, que ha dañado el mecanismo de mercado mediante la intervención directa en operaciones, y que ha renegado de sus compromisos establecidos en negociaciones bilaterales. Declara que no desea una guerra comercial, pero tampoco la teme, y que luchará si es necesario. Señala además que los problemas y disputas que se derivan de la creciente relación comercial entre China y Estados Unidos deben verse desde una perspectiva positiva, resolución de conflictos por medio de consultas y mecanismos de disputa en la Organización Mundial de Comercio. China, reitera, tiene abierta la puerta a las negociaciones (Information Office, 2018).

En septiembre, en una reunión del Consejo de Seguridad de la Organización de Naciones Unidas, Donald Trump acusó al gobierno chino de estar interviniendo para influir en los resultados de las elecciones de medio periodo de Estados Unidos, con el fin de favorecer a sus rivales del Partido Demócrata. No presentó pruebas que fundamentaran sus declaraciones. Dijo además que él era el primer presidente de Estados Unidos que enfrentaba a China de una manera contundente para que terminara con sus malas prácticas comerciales que afectaban al país. El ministro de Asuntos Exteriores de China, que estuvo presente en la reunión del Consejo de Seguridad, negó que su gobierno esté interviniendo en las elecciones de medio periodo de Estados Unidos. Wang dijo: "Nosotros no intervenimos en los asuntos internos de ningún país. No aceptamos las acusaciones contra China" (SCMP, 27 de septiembre de 2018).

En un discurso en el Hudson Institute, el vicepresidente de Estados Unidos, Mike Pence, afirmó que China está interfiriendo en los asuntos internos de su país. Con ello continúa la línea de hacer aparecer al gobierno chino como el culpable de desviar los resultados de las elecciones en favor de los demócratas.

\section{CHINA Y EL SUDESTE DE ASIA}

En abril se llevó a cabo la Cumbre Anual de la Asociación de Naciones del Sudeste de Asia (ANSEA) en la ciudad de Singapur. Durante sus discusiones evitaron hacer mención de los problemas generados con China a causa de las políticas seguidas por este país en el Mar del Sur de China y de la militarización de las islas artificiales. El 12 de junio, el ministro de Relaciones Exteriores de China, Wang Yi, le comentó al secretario de la ANSEA que trabajarían de manera conjunta para establecer el código de conducta en el Mar del Sur de 
China. En agosto se anunció que ya tenían un borrador del texto; no obstante, los líderes chinos siguen defendiendo su posición respecto a sus reclamos de soberanía en el área. El 27 de junio, el presidente Xi Jinping le comentó al secretario de Defensa de Estados Unidos, James Mattis, que China no permitiría ni siquiera la pérdida de una pulgada de territorio que reclamaba.

China y la ANSEA realizaron su primer ejercicio marítimo conjunto con el fin de profundizar la cooperación en los temas de defensa y seguridad. También, fuerzas de Malasia, China y Tailandia llevaron a cabo un ejercicio conjunto en dos Estados de Malasia y áreas colindantes, que concluyó el 29 de octubre (Global Times, 22 de octubre de 2018).

La relación de China con Malasia sufrió un cambio con la llegada al poder de Mahathir Mohamad, quien decidió parar el vínculo ferroviario de la Costa Este que tenía una inversión de 20 mil millones de dólares, además de dos oleoductos con 2.3 mil millones que estaban construyendo empresas chinas. El argumento fue que Malasia no podía pagar los costos; criticó a su antecesor y lo acuso de corrupción. En el mes de agosto, Mahathir fue a China en una visita de cinco días; se entrevistó con Xi Jinping y Li Keqiang, pero no mencionaron los proyectos cancelados. Mahathir, no obstante, declaró que los líderes chinos entendían el problema de deuda de Malasia. Las dos partes enfatizaron la cooperación sino-malaya.

La relación con Filipinas ha mejorado notablemente. En 2016 Filipinas llevó ante el tribunal internacional su conflicto territorial con China sobre las islas en disputa en el Mar del Sur de China. Pero ahora el presidente Rodrigo Duterte ha dejado atrás la disputa y ha aceptado la relación comercial y de inversión con China. El secretario de Asuntos Exteriores de Filipinas, Alan Peter Cayetano, realizó una visita a China en el mes de marzo. Wang Yi comentó que las relaciones sino-filipinas deben verse a la luz de la perspectiva estratégica de largo plazo, y profundizar la cooperación tratando los desacuerdos de manera apropiada.

Las relaciones entre China y Myanmar siguen plagadas de problemas. En junio y julio se realizaron reuniones de alto nivel entre funcionarios chinos y de Myanmar. En junio, Aung San Suu Kyi discutió con el ministro de Defensa chino Wei Fenghe sobre las relaciones bilaterales en materia de seguridad y estratégicas. Posteriormente Wang Yi se reunió con funcionarios de Myanmar para hablar sobre el proceso de diálogo para la paz y esfuerzos de reconciliación nacional. En el mes de julio, funcionarios de ambos lados dialogaron sobre la cooperación y cumplimiento de la ley en su frontera común. 
El gobierno de Myanmar decidió hacer una revisión de los proyectos de infraestructura con financiamiento chino, incluyendo el proyecto del Puerto en Kyaukpyu con un costo de 9 mil millones de dólares localizado en el Estado de Pakhine, hogar de los cientos de miles de desplazados Rohigya. A los funcionarios de Myanmar les preocupa el alto costo que implicaría una deuda de tal nivel para el país, pues al no poder pagarla tendrían que ceder derechos sobre el puerto a China. Para el gobierno chino es muy importante la construcción de este puerto, porque le permitiría vincularse a la ruta comercial del Océano Índico de manera directa, sin pasar por el Estrecho de Malaca, y porque podría diversificar sus rutas de transporte y abastecimiento de energía. (Sutter y Huang, 2018).

\section{Relaciones de China CON la Península Coreana}

China calificó la entrevista de Donald Trump y Kim Jong-un en Singapur como un paso más hacia la estabilización de la península coreana, al igual que la posición del presidente sudcoreano Moon Jae-in de esforzarse por lograr acuerdos y crear condiciones para el diálogo con el régimen del norte. Xi Jinping temía que China perdiera su lugar como árbitro. En la Declaración de Panmunjom, firmada entre Monn y Kim después de su encuentro el 27 de abril, se decía que habría reuniones trilaterales entre las dos Coreas y Estados Unidos; y cuadrilaterales luego de incluir a China. En marzo Xi llamó a Kim Jong-un a Beijing; en su reunión le comentó que China le daría ayuda económica y apoyo a los Kim (SCMP, 29 de marzo).

El líder norcoreano Kim Jong-un se reunió con Xi Jinping en el mes de mayo en la ciudad de Dalian, y en junio, en la ciudad de Beijing. Estas dos reuniones significaron el estrechamiento de su relación. En la reunión de Dalian, Xi hizo hincapié en la necesidad de restaurar la amistad tradicional de los dos países socialistas, el significado y la importancia de los intercambios de alto nivel, la restauración de la confianza mutua para salvaguardar intereses comunes. Para el presidente es muy importante que China no sea excluida de ningún proceso dirigido a cambiar la situación política de la península.

El presidente Trump no ha visto con buenos ojos el acercamiento de Kim Jong-Un con China. El 22 de mayo declaró que los líderes chinos habían ejercido su influencia para que Kim le demandara terminar con los ejercicios militares conjuntos con Corea del Sur a cambio de la desnuclearización. 


\section{CHINA-TAIWÁN}

China ha continuado su política de bloqueo hacia Taiwán en un intento por aislarla y presionarla para que su presidenta, Cai Yingwen, acepte el "principio de una sola China". Le ha quitado, además aliados diplomáticos. En mayo, la República Dominicana y Burkina Faso rompieron relaciones con Taiwán para establecerlas con China. El 21 de agosto Taiwán rompió relaciones con El Salvador, argumentando que le había pedido una gran suma y apoyo para el partido gobernante en la elección. El Salvador estableció relaciones con China.

También el gobierno chino presionó para que Taiwán no fuera apoyado por otros países para asistir como observador a la Asamblea de la Organización Mundial de la Salud. El gobierno chino advirtió a países que si apoyaban la participación de Taiwán en la asamblea podrían correr el riesgo de perder la cooperación con China. No obstante, el ministro de Salud de la isla, Chen Shizhong, viajó a Ginebra para conversar con otros representantes de manera informal. Además, el gobierno anunció que daría un donativo de un millón de dólares a la OMS para que combatiera el ébola.

A los socios no diplomáticos de Taiwán, el gobierno chino les pidió rebajar de nivel su relación con la isla. Por ejemplo, Nigeria le solicitó al gobierno taiwanés que moviera su oficina de representación de la capital de Abuja a Lagos. En Dubái, Ecuador, Bahrein, Jordania, Papúa Nueva Guinea, los funcionarios han sido presionados para que soliciten a los taiwaneses remover la palabra Taiwán o República de China en el nombre de la representación.

A finales de abril, Beijing hizo público un precepto dirigido a 44 líneas aéreas extranjeras en el que demandaba el cambio de nombre de Taiwán en sus páginas de internet, a "Taiwán, China". Puso como fecha límite el 25 de mayo. Dieciocho líneas aéreas cumplieron con lo solicitado, el resto pidió una prórroga hasta el 15 de julio. Por su parte, Estados Unidos pidió a sus líneas aéreas ignorar la demanda de China.

Al mismo tiempo, el gobierno chino hizo pública una lista de 31 incentivos para los taiwaneses residentes en China, de tal manera que se sientan parte del continente, con trabajos mejor pagados, acceso al mercado chino y trato igual. En el mes de agosto, Xi Jinping anunció que los habitantes de Hong Kong, Macao y Taiwán ya no necesitarían permisos de 
trabajo; a partir del 1 de septiembre serían elegibles para tener credenciales de residentes. (Brown y Churchman, 2018).

En agosto, la provincia de Fujian empezó a abastecer de agua a Jinmen mediante un ducto, con un contrato firmado por un periodo de 30 años.

Lian Zhan, líder del Guomindang de Taiwán, fue a China en el mes de julio y se entrevistó con Xi Jinping. En sus pláticas enfatizaron el “Consenso de 1992”.

Estados Unidos ha tratado de abrir la puerta para que los funcionarios taiwaneses puedan tener intercambios con sus contrapartes en el país. El 28 de febrero el Senado aprobó el Acta de Viajes de Taiwán que había sido ya aprobada por la Cámara de Representantes en el mes de enero. Estipula que Estados Unidos debe permitir que los funcionarios de todos los niveles viajen a Taiwán y puedan entrevistarse con sus contrapartes taiwaneses; también, que funcionarios taiwaneses de alto nivel entren al país, "Bajo condiciones de respeto" y se entrevisten con funcionarios estadounidenses (SCMP, 16 de marzo de 2018).

\section{CHINA-RUSIA}

Ante las acciones del presidente Trump, China y Rusia han fortalecido sus lazos en materia de seguridad y defensa, y presentado también un frente común enfocado a la cooperación y a intereses comunes. Putin fue a China en junio, después de ganar las elecciones y quedar como presidente. Xi y Putin acordaron expandir y profundizar la cooperación en todas las áreas. Definieron su relación como madura, estable y fuerte. En la Declaración conjunta señalan que la relación sino-rusa ejerce un papel importante en la preservación de la paz y la estabilidad mundial (Yu Bin, 2018).

Rusia llevó a cabo los ejercicios militares Vostok 2018 que iniciaron el 11 de septiembre, con 297 mil tropas, 36 mil vehículos y mil aeroplanos. De igual modo, China también participó enviando 3200 tropas, más de mil vehículos militares y equipo y 30 aeroplanos (SCMP, 12 de septembre de 2018). El Ejército de Liberación Nacional participó en ejercicios conjuntos con los rusos, simulando operaciones ofensivas y contraofensivas. Estos ejercicios militares simulan campañas de guerra. Existen cuatro motivaciones para que China participara en Vostok 2018: 1) Mostrar que China y Rusia están unidos ante las agresiones de Donald Trump; al igual que ante rivales chinos, como India y Vietnam; 2) 
mostrarle a Rusia los avances logrados con la reforma militar que permite que su ejército pueda tener un buen desempeño en caso de guerra; 3) también, observar qué otras reformas son necesarias dentro de su ejército, para hacer los ajustes pertinentes; 4) aprender de sus contrapartes rusos que han regresado después de participar en campañas en Ucrania del este y Siria.

Rusia y China están logrando dejar a un lado desavenencias del pasado para mostrar, sobre todo a Estados Unidos, que pueden formar un frente unido en caso de una situación de crisis (Zi Yang, 2018). China se ha convertido en el mayor socio comercial de Rusia y, desde la imposición de sanciones a este país, también en la mayor fuente de inversión extranjera; además de que ha incrementado su acceso a recursos energéticos rusos.

Finalmente, el acuerdo nuclear, negociado por más de dos años, quedó finalmente establecido. Hay tres proyectos que incluyen: la planta en Tianwan en Jiangsu, y la de Xudabao en Lioaning, que involucra una nueva plataforma y acceso a tecnología de punta rusa (SCMP, 8 de junio de 2018).

\section{CHINA-JAPÓN}

En mayo, el primer ministro Li Keqiang visitó Japón, la primera visita de este nivel en ocho años, para asistir a la reunión trilateral de jefes de gobierno de China, Japón y Corea del Sur. Los líderes de los tres países estuvieron de acuerdo en acelerar las negociaciones para un acuerdo de libre comercio entre ellos. Li sostuvo conversaciones separadas con Abe en las que acordaron establecer un mecanismo de vínculo tanto marítimo como aéreo para poder responder en forma conjunta a situaciones de crisis, de tal modo que el Mar del Sur de China sea lugar de cooperación, paz y seguridad. Antes, el 4 de mayo, el presidente Xi Jinping y el primer ministro Shinzo Abe hablaron por teléfono para comentar formas de mejorar la relación bilateral y para ponerse de acuerdo sobre la conmemoración de los 40 años de la firma del Tratado de Paz y Amistad China-Japón (Xinhua, 4 de mayo).

El 25 de octubre, el primer ministro Shinzo Abe llegó a China, la primera visita de este nivel desde 2011. Las pláticas Abe-Xi dieron como resultado la firma de más de 500 acuerdos de negocios con un valor total de más de 2.6 mil millones de dólares que van desde infraestructura, energía y proyectos de autos, hasta un pacto de intercambio de sus respectivas 
monedas por 30 mil millones de dólares. También discutieron sobre la necesidad de incrementar los intercambios de alto nivel, diplomáticos y militares. Abe señaló que su visita era un cambio histórico en las relaciones bilaterales que dejan a un lado la competencia y se centran en la colaboración. Xi Jinping señaló que las relaciones bilaterales debían moverse hacia una nueva dirección, en este mundo caracterizado por la inestabilidad. Los dos países tienen intereses comunes y preocupaciones compartidas (Xinjingbao, 2018).

Li Keqiang y Shinzo Abe enfatizaron la importancia del comercio libre, por lo que deben acelerarse las pláticas sobre el Acuerdo de Libre Comercio entre China, Japón y Corea del Sur. Además, durante la visita de Abe a China las compañías de los dos países firmaron acuerdos para llevar a cabo alrededor de 50 proyectos conjuntos en terceros países, como Tailandia. Abe señaló que en estos proyectos debe tenerse en cuenta la posición financiera de los países recipientes y seguir con los estándares internacionales (Mainichi, 26 de ctubre de 2018).

\section{FORO DE BOAO}

En abril se llevó a cabo la conferencia anual del Foro de Boao. El presidente Xi Jinping planteó la disyuntiva que se le presenta a la humanidad, de escoger entre la apertura o el aislamiento, entre progreso y regresión. La corriente actual, señaló, va en el sentido de paz y cooperación, apertura y conectividad. La globalización, dijo, es irreversible (Beijing Review, 2018). El presidente chino también anunció que su país adoptaba medidas para expandir la apertura, tales como mayor acceso a su mercado, protección de los derechos de propiedad intelectual, baja de tarifas.

\section{REUNiÓN ANUAL DE LA ORGANIZACIÓN DE COOPERACIÓN DE SHANGHÁI}

En el mes de junio se llevó a cabo la reunión número 18 de la Organización de Cooperación de Shanghái en la ciudad de Qingdao, China. En ella, Xi Jinping señaló que la OCS se ha convertido en una fuerza importante como promotora de la paz global y el desarrollo. El proteccionismo y el unilateralismo, dijo, se combaten con la interconexión y la cooperación para beneficio mutuo. Xi propuso cinco puntos: 1) Construir y fortalecer la confianza mutua; 2) Fortalecer el fundamento de la paz y la seguridad; 3) Construir la maquinaria para lograr 
el desarrollo y la prosperidad comunes; 4) Fomentar lazos cercanos mediante el intercambio cultural y de los pueblos; 5) Expandir redes de cooperación internacional (Beijing Review, 2018).

En esta reunión, India y Pakistán asistieron como miembros formales de la organización luego de completar los requisitos de su membresía. La OCS ya tiene ocho miembros: China, Rusia, Kazajistán, Kirguistán, Tayikistán, Uzbekistán, India, Pakistán que cubren el $60 \%$ del continente euroasiático, 34 millones de kilómetros cuadrados, $43 \%$ de la población global (que equivale a 3 mil millones) y más de $20 \%$ del PIB mundial (16 billones).

En la Declaración de Qingdao se señalan tres áreas específicas para la futura cooperación: seguridad, economía e intercambios culturales. Además de cumplir con sus tres metas originales: combate al terrorismo, extremismo y separatismo, también señalaron la coordinación en inteligencia, operaciones conjuntas contra tráfico de drogas, actividades criminales en sus fronteras y crimen cibernético. Putin informó que el gobierno sirio ya controlaba a $90 \%$ de la población, y por tanto los terroristas internacionales se irían moviendo hacia Afganistán.

En lo económico, los miembros se comprometieron a seguir el sistema comercial multilateral basado en el derecho. La declaración llama a una mayor cooperación en comercio, inversión, finanzas, agricultura y conectividad. En su discurso, Xi Jinping habló de la necesidad de cooperar en la promoción de la iniciativa de la Franja y la Ruta, y su vinculación con estrategias económicas de otros miembros de la OCS. Anunció la colocación de 30 mil millones de renminbi (4 mil millones de dólares) para préstamos dentro del marco del Consorcio Interbancario de la OCS. Qingdao será la ciudad para la cooperación económica y comercial China-OCS. Xi también anunció que otorgaría facilidades para el entrenamiento de recursos humanos de miembros de la OCS (SCMP, 8 de junio de 2018).

En agosto se llevaron a cabo los ejercicios anti-terroristas bianuales de la OCS, Misión de Paz 2018, en el distrito central militar de Rusia. Por primera vez participaron India y Pakistán. 


\section{CHINA Y LOS BRICS}

En julio se llevó a cabo la reunión de los BRICS (Brasil, Rusia, India, China y Sudáfrica) en la ciudad de Johannesburgo. El presidente Xi Jinping señaló que el mundo está experimentando una revolución en ciencia, tecnología e industria, profunda y extensa, con nueva tecnología y modelos de negocios. Pero los conflictos geopolíticos, la escalada proteccionista y unilateral afectan el ambiente de prosperidad de mercados emergentes y de países en desarrollo. Por ello los BRICS, continuó, deben profundizar la asociación estratégica, consolidar el marco de cooperación económica, política, de seguridad y de intercambios entre los pueblos. Xi propuso cuatro puntos: 1) Expandir la cooperación económica, comercial, financiera, de inversión y conectividad, esto es, trabajar para salvaguardar el régimen comercial multilateral, promover la liberalización comercial y de inversión, rechazar el proteccionismo; seguir con el desarrollo impulsado por la innovación y construir la asociación de los BRICS sobre la nueva revolución industrial para fortalecer la coordinación política y macroeconómica y encontrar mayor complementariedad en la estrategia de desarrollo, así como para y reforzar la competitividad de los BRICS; 2) Salvaguardar la paz y la seguridad globales, comprometidos con el multilateralismo; 3) Expandir el intercambio entre los pueblos; 4) Construir una red de asociaciones cercanas, cooperación de los BRICS dentro de la Organización de Naciones Unidas, el G20, para defender los intereses comunes (Beijing Review, 27 de julio de 2018).

\section{CONSIDERACIONES FINALES}

La restructuración administrativa llevada a cabo muestra la disposición del gobierno chino de agilizar los procesos de cambio que conlleven la realización de las metas planteadas, tanto en el aspecto político como en el económico. Ante los embates estadounidenses, la reacción debe ser rápida pero también contundente, teniendo en cuenta que las reacciones viscerales los ponen al mismo nivel que Trump. El liderazgo chino, con el proceso de centralización del poder en manos de Xi Jinping y el fortalecimiento del Partido Comunista Chino, desea proyectar una imagen de fuerza tanto dentro como fuera del país, lo mismo que su política exterior, dirigida hacia la distención de relaciones con Japón y una mayor cooperación con Rusia y sus vecinos del Sudeste de Asia. China sigue ocupando un lugar central en las 
reuniones de los organismos a los que pertenece, y marcando la pauta en cuanto a las políticas a seguir de manera conjunta.

\section{REFERENCIAS CONSULTADAS}

Beijing Review (2018), BFA Annual Conference Concludes with Globalization Consensus, 12 de abril. Disponible h: http://www. bjreview.com/World/201804/t20180412_800126764.html\#

Beijing Review (2018), Xi Calls for Building SCO Community with a Shared Future, 12 de junio.

Disponible

en: http://www.bjreview.com/World/201806/t20180610_80013247.html\#

Beijing Review (2018), Xi urges BRICS Countries to Deepen Strategic Partnership, Open up Second "Golden Decade". Disponible en: http://www.bjreview.com/Special_Report/2018/President_Xi_Visits_Arab_and_Afric an_Countries_Attends_BRICS_Summit/Latest_News/201807/t20180727_80013247.ht $\mathrm{ml} \#$

Bin Zhao (2018), China Economic Quarterly Q2b2018, PWC, China. Disponible en: http://www.pwcen.com/ceq

Brown, David y Kyle Churchman (2018), "China Taiwan Relations: Tsai Firm in the Face of Pressure", Comparative Connections, (20)2, pp. 61-70.

China Daily (2018), 26 de octubre.

Glasser, Bonnie y Kelly Flaherty (2018), "US-China Relations: Tit for Tat Tariffs", Comparative Connections", (20)2, septiembre, pp. 21-36.

Global Times (2018), 22 de octubre, pp. 1-2.

Information Office of the State Council, The People's Republic of China (2018), The Facts and China's. Position on China-US Trade Friction, septiembre.

Mainichi, 26 de octubre de 2018. Dispinible en: http://mainichi.jp/english/ $\operatorname{articles} / 20181026 / \mathrm{p} 2 \mathrm{a} / 00 \mathrm{~m} / \mathrm{Ona} / 032000 \mathrm{c}$ 
Naugthon, Barry (2018), “Xi’s System, Xi’s Men: After the March 2018 National People's Congress", China Leadership Monitor, núm. 56.

Naugthon, Barry (2018a), "Economic Policy under Trade War Conditions: Can China Move Beyond Tit for Tat?”, China Leadership Monitor, núm. 57.

Sutter, Robert y Chin-hao Huang (2018), "China Southeast Asia Relations: Assessing China's Expanding Influence amid Strong Countercurrents", Comparative Connections, (20)2, pp. 53-60.

South China Morning Post (2018), 16 de marzo. Disponible en: https://www.scmp.com/news/china/policies-politics/article/2137486/still-no-decisiontrump-signing-us-taiwan-travel-bill

South China Morning Post (2018), 20 de marzo, Disponible en: https://www.scmp.com/news/china/policies-politics/article/2137914/xi-jinpingsspeech-wrap-chinas-two-sessions-after

South China Morning Post (2018), 29 de marzo. Disponible en: http://www.scmp.com/news/china/diplomacydefence/article/2139355/kim-jong-untells-xi-jinping-hes-willing-denuclearise

South China Morning Post (2018), 8 de junio. Disponible en: https:/www.scmp.com/news/china/diplomacy-defence/article/2149991/xi-jinpingvladimir-putin-hail-all-time-high-ties-sign

South China Morning Post (2018), 27 de septiembre, Disponible en: http:www.scmp.com/news/world/united-states-canada/article/2165883/Donaldtrump-accuses-china-seeking-meddle-us

South China Morning Post (2018), 12 de septiembre. Disponible en: http://www. scmp.com/news/china/diplomacy/article/2163793/chinas-xi-jinping-russias-vladimirputin-agree-boost-ties-amid

Strait Times (2018), 17 de marzo. Disponible en: https://www.straitstimes.com/asia/eastasia/chinas-parliament-unanimously-re-elects-xi-jinping-as-president

World Bank Group (2018), China Economic Update, Report No. 126710-CN. 
Xinhua (2018), 13 de marzo. Disponible en: http://www.xinhuanet.com/english/2018-0313/c 137035291.htm

Xinhua (2018), 4 de mayo.

Xinjingbao, 新京报 (diario de Beijing) (2018), 26 de octubre.

Yu Bin (2018), "China-Russia Relations: Tales of Two Friends, Two Summits and Two Drills", Comparative Connections, (20)2, pp. 111-120.

Zi Yang, (2018), "Vostok 2018: Russia and China's Diverging Common Interests", The Diplomat, 17 de septiembre. 